\title{
An Asymptotic Approximation for a Type of Fourier Integral*
}

\author{
By Paul W. Schmidt
}

Abstract. A uniform asymptotic approximation which can be used for all $q h \geqslant 0$ is developed for the Fourier integral

$$
I(h)=\int_{q}^{z} \frac{f\left(\sqrt{y^{2}-q^{2}}\right)}{\left(y^{2}-q^{2}\right)^{1 / 2}} \sin y h d y
$$

under the assumptions that $h z>>1$, that the first $L+2$ derivatives of $f(y)$ are continuous for $0 \leqslant y \leqslant\left(z^{2}-q^{2}\right)^{1 / 2}$, and that the first $2 L+2$ derivatives of $f(y)$ are continuous at $y=0$.

The approximation is especially convenient when $z>>q$.

1. Introduction. Because of the importance of membrane research in many areas of biology, several $x$-ray techniques have been developed to study the structure of membranes. In some recent calculations [1] of the small angle $x$-ray scattering from suspensions of randomly oriented, independently scattering membranes, the expression for the small angle $x$-ray scattering from the membrane sample was found to require evaluation of an integral of the form

$$
I(h)=\int_{q}^{z} \frac{f\left(\sqrt{y^{2}-q^{2}}\right)}{\left(y^{2}-q^{2}\right)^{1 / 2}} \sin y h d y
$$

for all $q h \geqslant 0$. In (1), the assumptions are made that $h z>1$, that the first $L+2$ derivatives of the function $f(y)$ are continuous for $0 \leqslant y \leqslant\left(z^{2}-q^{2}\right)^{1 / 2}$, and that the first $2 L+2$ derivatives of $f(y)$ are continuous at $y=0$.

Integrals similar to (1) often occur in calculations of the intensity of the small angle $\mathrm{x}$-ray scattering for other particles for which one of the three dimensions characterizing the particles is much smaller than the other two. For example, in a computation of the small angle $\mathrm{x}$-ray scattering from randomly oriented platelets, the quantity $h M(h, x)$ has the form [2] of $I(h)$ in Eq. (1) above, with the function $f\left(\sqrt{y^{2}-q^{2}}\right)$ replaced by $\left(y^{2}-q^{2}\right)^{1 / 2} f\left(\sqrt{y^{2}-q^{2}}\right)$.

Erdélyi's asymptotic expansion [3] of Fourier integrals, even though applicable to (1), gives different expressions for $q=0$ and $q \neq 0$. When a single equation valid for all $q h$ is desired, another type of approximation, often called a uniform asymptotic expansion [4], is necessary. Bleistein [4] and Erdélyi [5] have obtained uniform asymptotic expansions of Laplace integrals.

Received August 30, 1976.

AMS (MOS) subject classifications (1970). Primary 41 A60, 42 A68.

* Work supported by the National Science Foundation.

Copyright (c) 1978, American Mathematical Society 
Below, by use of a partial integration technique similar to that employed by Bleistein [4], a uniform asymptotic expansion of the Fourier integral (1) is developed. This expansion can be used for all $q h \geqslant 0$ and is especially convenient when $z>>$.

In Section II, the lowest-order and second-lowest order approximations are calculated to illustrate the partial integration method used for the general expansion. This expansion is stated at the end of Section II. The detailed evaluation of the general expansion is outlined in Section III, with some of the longer calculations being given in the appendixes. (Readers not interested in the details of these calculations can omit Section III and the appendixes.) The error in the expansion and some other properties of the uniform asymptotic expansion are discussed in Section IV.

This approximation, which is based on the same method that was used in [2], is more convenient than the earlier expression. Also, a detailed analysis of the error of the new expansion is given which is applicable for all values of $q h$.

II. The Uniform Asymptotic Expansion of $I(h)$. The first term in the uniform asymptotic expansion can be found by writing (1) in the form

$$
I(h)=\int_{q}^{z} g_{0}\left(\sqrt{y^{2}-q^{2}}\right) \sin y h d y+f(0) \int_{q}^{z} \frac{\sin y h}{\left(y^{2}-q^{2}\right)^{1 / 2}} d y,
$$

where

$$
g_{0}(y)=\frac{f(y)-f(0)}{y} .
$$

By use of partial integration and the integral representation [6]

$$
J_{0}(q x)=\frac{2}{\pi} \int_{q}^{\infty} \frac{\sin (x t)}{\left(t^{2}-q^{2}\right)^{1 / 2}} d t
$$

for $J_{0}(q x)$, the Bessel function of the first kind and order zero, $I(h)$ can be expressed

$$
I(h)=B_{0}(h)-A_{0}(h)+R_{0}(h)
$$

where

$$
\begin{aligned}
& A_{0}(h)=-\frac{\pi}{2} f(0) J_{0}(q h)-f^{(1)}(0) \frac{\cos q h}{h}, \\
& B_{0}(h)=-\frac{f\left(\sqrt{z^{2}-q^{2}}\right)}{\left(z^{2}-q^{2}\right)^{1 / 2}} \frac{\cos z h}{h}, \\
& R_{0}(h)=S_{0}(h)+T_{0}(h), \\
& S_{0}(h)=f(0) \int_{z}^{\infty} \frac{y}{\left(y^{2}-q^{2}\right)^{3 / 2}} \frac{\cos y h}{h} d y, \\
& T_{0}(h)=\int_{q}^{z} y \frac{g_{0}^{(1)}\left(\sqrt{y^{2}-q^{2}}\right)}{\left(y^{2}-q^{2}\right)^{1 / 2}} \frac{\cos y h}{h} d y,
\end{aligned}
$$




$$
f^{(1)}(y)=\frac{d f}{d y}, \quad g_{0}^{(1)}(y)=\frac{d g_{0}}{d y} .
$$

The lowest-order approximation for $I(q)$ is obtained by neglecting the remain$\operatorname{der} \boldsymbol{R}_{\mathbf{0}}(h)$. According to the properties of asymptotic expansions of Fourier integrals [3], [7],

$$
R_{0}(h)=o\left(h^{-1}\right) \quad(h \rightarrow \infty)
$$

It is important to note that this approximation can be used for all values of $q$ h.

To obtain the next term in the uniform asymptotic expansion of (1), the integral $T_{0}(h)$ in (2) can be written

$$
T_{0}(h)=i_{1}(h)+2^{-1 / 2} \Gamma(1 / 2) \int_{q}^{z} y^{2} g_{1}\left(\sqrt{y^{2}-q^{2}}\right) k_{0}(y h) d y,
$$

where

$$
\begin{aligned}
i_{1}(h) & =2^{-1 / 2} \Gamma(1 / 2) g_{0}^{(1)}(0) \int_{q}^{z} y^{2} \frac{k_{0}(y h)}{\left(y^{2}-q^{2}\right)^{1 / 2}} d y \\
& =\frac{f^{(2)}(0)}{2 h} \frac{d}{d h} \int_{q}^{z} \frac{\sin h y}{\left(y^{2}-q^{2}\right)^{1 / 2}} d y \\
& =\frac{f^{(2)}(0)}{2 h} \frac{d}{d h}\left[\frac{\pi}{2} J_{0}(q h)-\int_{z}^{\infty} \frac{\sin q h}{\left(y^{2}-q^{2}\right)^{1 / 2}} d y\right] \\
k_{\alpha}(x) & =x^{-(\alpha+1 / 2)} J_{-(\alpha+1 / 2)}(x), \quad g_{1}(x)=\frac{g_{0}^{(1)}(x)-g_{0}^{(1)}(0)}{x} .
\end{aligned}
$$

Since the Bessel functions have the property that [8]

$$
\frac{d}{d x}\left[x^{-\alpha} J_{\alpha}(x)\right]=-x^{-\alpha} J_{\alpha+1}(x),
$$

by partial integration, $T_{0}(h)$ can be written

$$
T_{0}(h)=i_{1}(h)+Y_{1}(h)+T_{1}(h),
$$

where

$$
Y_{1}(h)=-2^{-1 / 2} \Gamma(1 / 2)\left[z^{3} g_{1}\left(\sqrt{z^{2}-q^{2}}\right) k_{1}(z h)-q^{3} g_{1}(0) k_{1}(q h)\right]
$$

and

$$
T_{1}(h)=2^{-1 / 2} \Gamma(1 / 2) \int_{q}^{z} y^{4} g_{1}^{(1)}\left(\sqrt{y^{2}-q^{2}}\right) \frac{k_{1}(h y)}{\left(y^{2}-q^{2}\right)^{1 / 2}} d y .
$$

To permit evaluation of the derivative in $i_{1}(h)$, the integral can be integrated by parts to give

$$
i_{1}(h)=\frac{f^{(2)}(0)}{2 h} \frac{d}{d h}\left[\frac{\pi}{2} J_{0}(q h)-\sqrt{\frac{\pi}{2}} \frac{z}{\left(z^{2}-q^{2}\right)^{1 / 2}} k_{0}(h z)+\frac{1}{h} \int_{z}^{\infty} \frac{y \cos h y}{\left(y^{2}-q^{2}\right)^{3 / 2}} d y\right] .
$$


Therefore,

$$
i_{1}(h)=\sqrt{\frac{\pi}{2}} \frac{f^{(2)}(0)}{2}\left[-\sqrt{\frac{\pi}{2}} q^{2} \frac{J_{1}(q h)}{q h}-\frac{z^{3} k_{1}(z h)}{\left(z^{2}-q^{2}\right)^{1 / 2}}+\int_{z}^{\infty} \frac{y^{4} k_{1}(y h)}{\left(y^{2}-q^{2}\right)^{3 / 2}} d y\right] .
$$

By partial integration, the integral $S_{0}(h)$ defined in (2) can be expressed

$$
S_{0}(h)=\sqrt{\frac{\pi}{2}} f(0)\left[\frac{z^{3} k_{1}(z h)}{\left(z^{2}-q^{2}\right)^{3 / 2}}-3 \int_{z}^{\infty} \frac{y^{4} k_{1}(y h)}{\left(y^{2}-q^{2}\right)^{5 / 2}} d y\right] \text {. }
$$

With (2) and the above expressions for $S_{0}(h)$ and $T_{0}(h)$,

$$
\begin{aligned}
I(h) & =B_{0}(h)-A_{0}(h)+S_{0}(h)+T_{0}(h) \\
& =B_{1}(h)-A_{1}(h)+R_{1}(h)
\end{aligned}
$$

where

$$
\begin{aligned}
& A_{1}(h)=-\frac{\pi}{2} \sum_{j=0}^{3} \frac{f^{(j)}(0) q^{j}}{2^{j / 2} \Gamma\left(\frac{j}{2}+1\right)} k_{(j-1) / 2}(q h), \\
& B_{1}(h)=-2^{-1 / 2} \Gamma\left(\frac{1}{2}\right) \sum_{j=0}^{1} z^{2 j+1} e_{j}\left(\sqrt{z^{2}-q^{2}}\right) k_{j}(z h), \\
& R_{1}(h)=S_{1}(h)+T_{1}(h), \\
& S_{1}(h)=-\sqrt{\frac{\pi}{2}} \int_{z}^{\infty}\left[3 f(0)-\frac{f^{(2)}(0)}{2}\left(y^{2}-q^{2}\right)\right] \frac{y^{4} k_{1}(y h)}{\left(y^{2}-q^{2}\right)^{5 / 2}} d y, \\
& T_{1}(h)=\sqrt{\frac{\pi}{2}} \int_{q}^{z} y^{4} g_{1}^{(1)}\left(\sqrt{y^{2}-q^{2}}\right) \frac{k_{1}(y h)}{\left(y^{2}-q^{2}\right)^{1 / 2}} d y, \\
& e_{0}(x)=\frac{f(x)}{x}, \quad e_{j+1}(x)=\frac{1}{x} \frac{d}{d x}\left[e_{j}(x)\right] .
\end{aligned}
$$

The second asymptotic approximation for $I(h)$ is obtained by letting

$$
I(h) \simeq B_{1}(h)-A_{1}(h) .
$$

That is, the remainder $R_{1}(h)$ is neglected.

The technique used to find this asymptotic approximation can be extended to obtain the general uniform asymptotic expansion for $I(h)$. As is shown in Section III,

$$
I(h)=I_{L}(h)+R_{L}(h) .
$$

The $L$ th-order approximation $I_{L}(h)$ and the remainder $R_{L}(h)$ are defined in Eqs. (4)-(9).

In the $L$ th-order approximation

$$
I(h) \sim I_{L}(h) .
$$


That is, the remainder $R_{L}(h)$ is neglected. As is explained below,

$$
R_{L}(h)=o\left[h^{-(L+1)}\right] \quad(h \rightarrow \infty) .
$$

Eqs. (3)-(9) state but do not prove the general approximation, the detailed development of which is outlined in Section III. Some of the longer calculations are given in the appendixes.

The $L$ th-order approximation can be written

$$
I_{L}(h)=B_{L}(h)-A_{L}(h)
$$

where

$$
\begin{aligned}
& A_{L}(h)=-\frac{\pi}{2} \sum_{j=0}^{2 L+1} m_{j / 2}(q) k_{(j-1) / 2}(q h) \\
& B_{L}(h)=-2^{-1 / 2} \Gamma(1 / 2) \sum_{j=0}^{L} z^{2 j+1} e_{j}\left(\sqrt{z^{2}-q^{2}}\right) k_{j}(z h),
\end{aligned}
$$

(4)

$$
\begin{aligned}
& m_{\alpha}(x)=\frac{f^{(2 \alpha)}(0) x^{2 \alpha}}{2^{\alpha} \Gamma(\alpha+1)}, \quad k_{j}(x)=x^{-j-1 / 2} J_{-j-1 / 2}(x), \\
& e_{0}(x)=\frac{f(x)}{x}, \quad e_{j+1}(x)=\frac{1}{x} \frac{d}{d x}\left[e_{j}(x)\right], \quad j \geqslant 0 .
\end{aligned}
$$

In (4), for any function $F(x)$,

$$
F^{(j)}(x)=d^{j} F / d x^{j}
$$

$J_{\alpha}(x)$ is the Bessel function of the first kind and order $\alpha$, and $\Gamma(x)$ is the gamma function.

The remainder $R_{L}(h)$ then is given by the expression

$$
R_{L}(h)=S_{L}(h)+T_{L}(h)
$$

where

$$
S_{L}(h)=-\sum_{j=0}^{j \leqslant(L+1) / 2} v_{L-j, j}(h)-\sum_{j>(L+1) / 2}^{L}\left[m_{j}(z) u_{L+1-j, L+1}^{2 j-2-L}(h)+v_{j-1, j}(h)\right],
$$

$$
\begin{gathered}
u_{i j}^{L}(h)=\sum_{n=0}^{L} h_{i+n}(z) k_{j+n}(z h), \\
v_{i j}(h)=\int_{z}^{\infty} m_{j}(y) h_{i+1}(y) k_{i+j}(y h) \frac{d y}{y}, \\
h_{i}(x)=(-1)^{i} 2^{i-1 / 2} \Gamma(i+1 / 2) x^{2 i+1}\left(x^{2}-q^{2}\right)^{-i-1 / 2},
\end{gathered}
$$

$$
T_{L}(h)=\int_{q}^{z} y^{2 L+2} g_{L}^{(1)}\left(\sqrt{y^{2}-q^{2}}\right) h_{0}(y) k_{L}(y h) \frac{d y}{y}
$$




$$
g_{0}(x)=\frac{f(x)-f(0)}{x}
$$

(9)

$$
g_{j+1}(x)=\frac{g_{j}^{(1)}(x)-g_{j}^{(1)}(0)}{x}, \quad j \geqslant 0 \text {. }
$$

In the definition of $S_{L}(h)$ in (7), the sum is defined to be equal to zero if $(L+1) / 2$ $\leqslant L$-that is, if $L \leqslant 1$. The integral in the expression for the $v_{i j}$ in (7) converges if $i \geqslant j-1$.

For $L=0$, (3) reduces to (2).

III. Calculation of the General Uniform Asymptotic Expansion. With the quantities defined in Eqs. (4)-(9), (3) can be verified by induction. The first step in this calculation is to rewrite $T_{L}(h)$, which is defined in (8), to give

$$
T_{L}(h)=i_{L+1}(h)+2^{-1 / 2} \Gamma(1 / 2) \int_{q}^{z} y^{2 L+2} g_{L+1}\left(\sqrt{y^{2}-q^{2}}\right) k_{L}(y h) d y
$$

where

$$
i_{L+1}(h)=g_{L}^{(1)}(0) \int_{q}^{z} y^{2 L+2} h_{0}(y) k_{L}(y h) \frac{d y}{y} .
$$

When $g_{L}^{(1)}(0)$ is evaluated with $(\mathrm{AI}-2), i_{L+1}(h)$ can be written

$$
i_{L+1}(h)=\int_{q}^{z} m_{L+1}(y) h_{0}(y) k_{L}(y h) \frac{d y}{y} .
$$

The integral in (10) can be integrated by parts by use of (AII-8). Then

$$
T_{L}(h)=i_{L+1}(h)+Y_{L+1}(h)+T_{L+1}(h)
$$

where

$$
Y_{L+1}(h)=-\frac{\Gamma(1 / 2)}{2^{1 / 2}}\left[z^{2 L+3} g_{L+1}\left(\sqrt{z^{2}-q^{2}}\right) k_{L+1}(z h)-q^{2 L+3} g_{L+1}(0) k_{L+1}(q h)\right] \text {. }
$$

Substitution of (AII-11), (AI-2), (AI-4), and (AI-7) in the above expressions for $T_{L}(h)$ and $Y_{L+1}(h)$ gives

(12) $T_{L}(h)=-A_{L+1}(h)+A_{L}(h)+B_{L+1}(h)-B_{L}(h)+T_{L+1}(h)+X_{L+1}(h)$,

$$
\begin{gathered}
X_{L+1}(h)=-m_{L+1}(z) u_{0, L+1}^{L}(h)-v_{L, L+1}(h)+w_{L+1}(z) k_{L+1}(z h) \\
w_{L}(z)=\sum_{i=0}^{L} m_{i}(z) h_{L-i}(z)
\end{gathered}
$$

From the definition of the $u_{i j}^{L}(h)$ given in (7),

$$
u_{L+1-j, L+1}^{2 j-2-L}(h)=u_{L+2-j, L+2}^{2 j-3-L}(h)+h_{L+1, j}(h) k_{L+1}(h) .
$$

In (14), $u_{i j}^{L}(h)$ is defined to equal zero unless $L \geqslant 0$. When (AII-8) is used to perform 
a partial integration in the equation for the $v_{i j}(h)$ in (7), the expression

$$
v_{L-j, j}(h)=v_{L+1-j, j}(h)+m_{j}(z) h_{L+1-j}(z) k_{L+1}(z h)
$$

is obtained.

As can be verified by substitution,

$$
\begin{aligned}
\sum_{j=0}^{j \leqslant(L+1) / 2} v_{L+1-j, j}(h) & +\sum_{j>(L+1) / 2}^{L+1} v_{j-1, j}(h) \\
& =\sum_{j=0}^{j \leqslant(L+2) / 2} v_{L+1-j, j}(h)+\sum_{j>(L+2) / 2}^{L+1} v_{j-1, j}(h) .
\end{aligned}
$$

From the definition of $S_{L}(h)$ given in (7), and from (14), (15), (16), and the convention assumed for the $u_{i j}^{L}(h)$ in (14),

$$
S_{L}(h)=-X_{L+1}(h)+S_{L+1}(h) .
$$

Since $R_{L}(h)=T_{L}(h)+S_{L}(h)$, from (12) and (17),

$$
A_{L}(h)+B_{L}(h)+R_{L}(h)=A_{L+1}(h)+B_{L+1}(h)+R_{L+1}(h) .
$$

Eq. (18) can be used to prove (3) by induction, since $I(h)=A_{0}(h)+B_{0}(h)+R_{0}(h)$.

IV. Discussion. From the properties of asymptotic expansions of Fourier integrals [3], [7]

$$
R_{L}(h)=o\left[h^{-(L+1)}\right] \quad(h \rightarrow \infty) .
$$

Eq. (3) is most useful when $B_{L}(h)$ is either relatively small or completely negligible. The principal contribution to $I(h)$ then comes from $A_{L}(h)$.

The approximation developed in [2] contains a sum equivalent to $A_{L}(h)$. As was mentioned in the introduction, the approximation (3) given here is a more convenient and explicit expression for the error than was provided by the earlier result in [2]. In addition, $\boldsymbol{B}_{L}(h)$ is usually more convenient to use than the corresponding sum given in [2].

The sum $A_{L}(h)$ is the feature that distinguishes the approximation (3) from the conventional asymptotic expansion for Fourier integrals. The important property of (3) is that it can be used for all values of $q h$. As $q h$ approaches 0 , the limiting expression for $A_{L}(h)$ is

$$
-A_{L}(h) \simeq \frac{\pi}{2}+\sum_{j=0}^{L} \frac{(-1)^{j} f^{(2 j+1)}(0)}{(2 j+1) h^{2 j+1}} .
$$

The form of $A_{L}(h)$ for large $q h$ can be found by substituting the asymptotic expansions for the Bessel functions in the expression for $A_{L}(h)$. The behavior of $A_{L}(h)$ is seen to be different for large and small $q h$.

According to Eqs. (AIII-5) and (AIII-6) of Appendix III, $g_{L}(x)$ and $g_{L}^{(1)}(x)$ can be written 


$$
g_{j}(x)=\sum_{i=0}^{j} \frac{(-1)^{j-1}(2 j-i) ! F_{i j}(x)}{2^{j-i} i !(j-i) ! x^{2 j+1-i}}
$$

and

$$
g_{j}^{(1)}(x)=\sum_{i=0}^{j+1} \frac{(-1)^{j+1-i}(2 j+2-i) ! F_{i j}(x)}{2^{j+1-i} i !(j+1-i) ! x^{2 j+2-i}}
$$

where, for $j \geqslant 1$ and also for $i=j=0$,

$$
F_{i j}(x)=f^{(i)}(x)-\sum_{k=0}^{2 j-i} \frac{f^{(i+k)}(0) x^{k}}{k !},
$$

and where $F_{10}(x)=f^{(1)}(x)$. When $2 j-i \geqslant 0$, the $F_{i j}(x)$ are the difference between $f^{(i)}(x)$ and the Taylor expansion of $f^{(i)}(x)$ of order $2 j-i$ about the point $x=0$.

The magnitude of $g_{L}^{(1)}(x)$ will usually be smaller than would be immediately apparent from the magnitudes of the individual terms in the expression for $g_{L}^{(1)}(x)$ obtained directly from (AI-3) and (AI-6), since, as (20) shows, $g_{L}^{(1)}(x)$ can be written as a sum of the $F_{i j}(x)$, each of which, except $F_{10}(x)$, is the difference of $f^{(i)}(x)$ and a Taylor series approximation for $f^{(i)}(x)$. Ordinarily, this difference can be expected to be smaller in magnitude than $f^{(i)}(x)$ itself.

This result is useful in setting bounds on the remainder $T_{L}(h)$ defined in (8).

If $f(x)$ is a polynomial of degree $m$, the $F_{i L}(x)$ will be zero if $2 L \geqslant m$; and consequently, $T_{L}(h)$ will be zero for $L \geqslant m / 2$, and from (6), the remainder $R_{L}(h)$ will be equal to $S_{L}(h)$.

\section{APPENDIX I}

The functions $g_{j}(x), e_{j}(x)$, and $d_{j}(x)$. Since the first $2 L+2$ derivatives of $f(x)$ are assumed to be continuous at $x=0, f(x)$ can be approximated by the Taylor series

$$
f(x) \simeq \sum_{j=0}^{2 L+2} \frac{f^{(j)}(0)}{j !} x^{j}
$$

in the neighborhood of $x=0$.

The corresponding series approximation for the $g_{j}(x)$ defined in (9) is

$$
g_{j}(x) \simeq \sum_{i=0}^{2 L+1-2 j} \frac{f^{(2 j+1+i)}(0) \Gamma\left(\frac{i+1}{2}\right)}{2^{j+1} i ! \Gamma\left(\frac{i+2 j+3}{2}\right)} x^{i}
$$

This expression satisfies (9) with the same accuracy as the series for $f(x)$.

From (AI-1)

$$
g_{j}(0)=\frac{\Gamma(1 / 2) f^{(2 j+1)}(0)}{2^{j+1} \Gamma\left(j+\frac{3}{2}\right)}
$$




$$
g_{j}^{(1)}(0)=\frac{f^{(2 j+2)}(0)}{2^{j+1}(j+1) !}
$$

As can be verified by substitution in (4), the $e_{j}(x)$ are given exactly by

$$
e_{j}(x)=\sum_{i=0}^{j} \frac{(-1)^{j-i}(2 j-i) ! f^{(i)}(x)}{2^{j-i} i !(j-i) ! x^{2 j+1-i}}
$$

Let

$$
d_{j}(x)=e_{j}(x)-g_{j}(x)
$$

Then, from (4) and (9), $d_{0}(x)=f(0) / x$ and for $j \geqslant 0$,

$$
d_{j+1}(x)=\frac{d_{j}^{(1)}(x)}{x}+\frac{g_{j}^{(1)}(0)}{x}
$$

From (AI-5), the exact expression for the $d_{j}(x)$ can be shown to be

$$
d_{j}(x)=\sum_{i=0}^{j} \frac{(-1)^{j-i} 2^{j-2 i} \Gamma(j-i+1 / 2) f^{(2 i)}(0)}{i ! \Gamma(1 / 2) x^{2 j+1-2 i}}
$$

An exact equation for the $g_{j}(x)$ can be obtained from (AI-3), (AI-4) and (AI-6). With (4) and (7), (AI-6) gives

$$
2^{-1 / 2} \Gamma(1 / 2) y^{2 j+1} d_{j}\left(\sqrt{y^{2}-q^{2}}\right)=\sum_{i=0}^{j} m_{i}(y) h_{j-i}(y) .
$$

\section{APPENDIX II}

Evaluation of $i_{j}(h)$. As in (11), let

$$
i_{j}(h)=\int_{q}^{z} m_{j}(y) h_{0}(y) k_{j-1}(y h) \frac{d y}{y} .
$$

For a function $f(x)$, let the operator $H$ be defined to have the property that

$$
H f(h)=\frac{1}{h} \frac{d f}{d h} .
$$

Then, as can be shown by induction, if $H^{n}$ denotes $n$ successive applications of $H$,

$$
H^{n} k_{j}(x h)=x^{2 n} k_{j+n}(x h),
$$

since [8] the Bessel functions have the property that

$$
\frac{d}{d x} x^{\alpha} J_{\alpha}(x)=x^{\alpha} J_{\alpha-1}(x)
$$

Thus,

$$
i_{j}(h)=\frac{f^{(2 j)}(0)}{2^{j} j !} H^{j}\left[\frac{\pi}{2} J_{0}(q h)-t_{00}(h)\right],
$$


where for $i \geqslant 0$ and $0 \leqslant j \leqslant i$,

$$
t_{i j}(h)=\int_{z}^{\infty} y^{2 j} h_{i}(y) k_{i+j-1}(y h) \frac{d y}{y}
$$

Equation (AII-5) is obtained by making use of the integral representation [6] which, in the notation used here, can be written

$$
\frac{\pi}{2} J_{0}(q h)=\int_{q}^{\infty} h_{0}(y) k_{-1}(y h) \frac{d y}{y} .
$$

The condition $j \leqslant i$ ensures the convergence of the integral in (AII-6).

Since [8]

$$
\frac{d}{d x}\left[x^{-\alpha} J_{\alpha}(x)\right]=-x^{-\alpha} J_{\alpha+1}(x),
$$

the $k_{j}(x)$ have the property that

$$
\int y^{2 j+2} k_{j}(y h) d y=-y^{2 j+3} k_{j+1}(y h) .
$$

Thus, $N+1$ integrations by parts give

$$
\begin{aligned}
t_{i j}(h) & =z^{2 j} \sum_{n=0}^{N} h_{i+n}(z) k_{i+j+n}(z h)+t_{i+N+1, j}(h) \\
& =z^{2 j} u_{i, i+j}^{N}(h)+t_{i+N+1, j}(h),
\end{aligned}
$$

where the $u_{i j}^{N}(h)$ are defined in (7). For $N=j-1$,

$$
H^{j} t_{00}(h)=z^{2 j} u_{0 j}^{j-1}(h)+t_{j j}(h) \text {. }
$$

Since $k_{n-1 / 2}(q h)=(q h)^{-n} J_{-n}(q h)$,

$$
H^{n} J_{0}(q h)=q^{2 n}(q h)^{-n} J_{-n}(q h)=q^{2 n} k_{n-1 / 2}(q h)
$$

Substitution of (AII-9) and (AII-10) in (AII-5) gives

$$
i_{j}(h)=\frac{\pi}{2} m_{j}(q) k_{j-1 / 2}(q h)-m_{j}(z) u_{0 j}^{j-1}(h)-v_{j-1, j}(h) .
$$

\section{APPENDIX III}

Rearrangement of the Expressions for $g_{j}(x)$ and $g_{j}^{(1)}(x)$. With (AI-3), the $e_{j}(x)$ can be expressed

$$
e_{j}(x)=\sum_{i=0}^{j} \frac{(-1)^{j-i}(2 j-i) ! F_{i j}(x)}{2^{j-i} i !(j-i) ! x^{2 j+1-i}}+D_{j}(x),
$$

where for $0 \leqslant i \leqslant j$

$$
F_{i j}(x)=f^{(i)}(x)-\sum_{k=0}^{2 j-i} \frac{f^{(i+k)}(0) x^{k}}{k !},
$$


(AIII-3)

$$
D_{j}(x)=\sum_{i=0}^{j} \frac{(-1)^{j-i}(2 j-i) !}{2^{j-i} i !(j-i) ! x^{2 j+1-i}} \sum_{k=0}^{2 j-i} \frac{f^{(i+k)}(0) x^{k}}{k !} .
$$

By interchange of the order of summation, (AIII-3) can be written

$$
D_{j}(x)=\sum_{i=0}^{2 j} \frac{d_{i j} f^{(i)}(0)}{x^{2 j+1-i}}
$$

where

$$
\begin{gathered}
d_{i j}=\sum_{k=0}^{k_{m}} \frac{(-1)^{j-k}(2 j-k) !}{2^{j-k} k !(j-k) !(i-k) !}, \\
k_{m}=j, \quad j \leqslant i, \\
k_{m}=i, \quad j \geqslant i .
\end{gathered}
$$

When the value of $d_{i j}$ given in Eq. (AIV-3) of Appendix IV is substituted in (AIII-4), the $D_{j}(x)$ are seen to be identical to the $d_{j}(x)$ given in (AI-6). Thus, from (AI-4),

$$
g_{j}(x)=\sum_{i=0}^{j} \frac{(-1)^{j-i}(2 j-i) ! F_{i j}(x)}{2^{j-i} i !(j-i) ! x^{2 j+1-i}} .
$$

Differentiation of (AIII-2) gives

$$
\left[F_{i j}(x)\right]^{(1)}=F_{i+1, j}(x) .
$$

From this result and (AIII-5),

(AIII-6)

$$
g_{j}^{(1)}(x)=\sum_{i=0}^{j+1} \frac{(-i)^{j+1-i}(2 j+2-i) ! F_{i j}(x)}{2^{j+1-i} i !(j+1-i) ! x^{2 j+2-i}},
$$

where $F_{10}(x)=f^{(1)}(x)$, and the other $F_{i j}(x)$ are given by (AIII-2).

\section{APPENDIX IV}

Evaluation of the $d_{i j}$. Let

$$
Q_{j}(y)=\left(1-y^{2}\right)^{j}
$$

Then

$$
Q_{j}^{(2 j-i)}(0)=\left.\sum_{k=0}^{j} \frac{(-1)^{k} j !}{k !(j-k) !}\left[y^{2 k}\right]^{(2 j-1)}\right|_{y=0}
$$

(AIV-1)

$$
=\frac{(-1)^{j-1 / 2 i} j !(2 j-i) !}{(j-1 / 2 i) !(1 / 2 i) !}
$$

for even $i$, while $Q_{j}^{(2 j-i)}(0)=0$ for odd $i$.

Also, if $y=2 x-1$,

$$
Q_{j}(y)=2^{2 j} x^{j}(1-x)^{j}=2^{2 j} q_{j}(x),
$$


where

$$
q_{j}(x)=x^{j}(1-x)^{j}
$$

If superscripts in parentheses indicate derivatives of a function with respect to its argument,

$$
Q_{j}^{(2 j-i)}(0)=2^{2 j}\left[q_{j}(1 / 2)\right]^{(2 j-i)}(1 / 2)^{2 j-i}=2^{i}\left[q_{j}(1 / 2)\right]^{(2 j-i)}
$$

(AIV-2)

$$
\begin{aligned}
& =\left.2^{i} \sum_{k=0}^{j} \frac{(-1)^{j-k} j !}{k !(j-k) !}\left[x^{2 j-k}\right]^{(2 j-i)}\right|_{x=1 / 2} \\
& =2^{j} d_{i j}
\end{aligned}
$$

where the $d_{i j}$ are defined below (AIII-4).

Thus, from (AIV-1) and (AIV-2),

$$
d_{2 k, j}=\frac{(-1)^{j-k} 2^{j-2 k} \Gamma(j-k+1 / 2)}{k ! \Gamma(1 / 2)}, \quad d_{2 k+1, j}=0,
$$

where $k$ is a nonnegative integer.

Acknowledgement. I thank Brian DeFacio for advice and assistance in the preparation of the manuscript.

Physics Department

University of Missouri

Columbia, Missouri 65201

1. H. WU \& P. W. SCHMIDT, research to be published.

2. P. W. SCHMIDT, J. Math. Phys., v. 7, 1966, p. 1295.

3. A. ERDÉLYI, Asymptotic Expansions, Dover, New York, 1956, pp. 49-50.

4. N. BLEISTEIN, Comm. Pure Appl. Math., v. 19, 1966, p. 353.

5. A. ERDÉLYI, SIAM J. Math. Anal., v. 5, 1974, p. 159.

6. W. MAGNUS \& F. OBERHETTINGER, Formulas and Theorems for the Functions of Mathematical Physics, Chelsea, New York, 1954, p. 27.

7. F. W. J. OLVER, Asymptotics and Special Functions, Academic Press, New York, 1974, pp. 4-6.

8. Reference 6, p. 16, Equations (2a) and (2b). 